\title{
Predictive power of the ADHD GWAS 2019 polygenic risk scores in independent samples of bipolar patients with childhood ADHD
}

Maria Grigoroiu-Serbanescu ${ }^{1}$, Giovanni Giaroli ${ }^{2}$, Johan H. Thygesen ${ }^{2}$, Oris Shenyan², Tim Bigdeli ${ }^{3}$, Nicholas J. Bass ${ }^{2}$, Carmen C. Diaconu ${ }^{4}$, Ana Iulia Neagu ${ }^{4}$, Andreas J. Forstner ${ }^{5,6,7}$, Franziska Degenhardt ${ }^{5}$, Stefan Herms ${ }^{5,7}$, Markus M. Nöthen ${ }^{5}$, Andrew McQuillin ${ }^{2}$

${ }^{1}$ Psychiatric Genetics Research Unit, Alexandru Obregia Clinical Psychiatric Hospital, Bucharest, Romania

${ }^{2}$ Molecular Psychiatry Laboratory, Division of Psychiatry, University College London, London, UK

${ }^{3}$ SUNY Downstate Medical Center, Brooklyn, New York, USA

${ }^{4}$ Stefan S Nicolau Institute of Virology, Bucharest, Romania

${ }^{5}$ Institute of Human Genetics, University of Bonn, School of Medicine \& University Hospital Bonn, Bonn, Germany

${ }^{6}$ Centre for Human Genetics, University of Marburg, Marburg, Germany

${ }^{7}$ Department of Biomedicine, University of Basel, Basel, Switzerland

\section{Corresponding authors:}

Maria Grigoroiu-Serbanescu, PhD, Psychiatric Genetics Research Unit, Alexandru Obregia Clinical Psychiatric Hospital, Bucharest, Romania. e-mail: maria.serbanescu@googlemail.com. Andrew McQuillin, Molecular Psychiatry Laboratory, Division of Psychiatry, University College London, London, UK. E-mail: a.mcquillin@ucl.ac.uk

Running head: ADHD polygenic risk score in bipolar disorder 


\section{Abstract (Version 1 for the main manuscript) (253 words)}

Background: Although there is evidence of genetic correlation between bipolar disorder (BP) and ADHD, the extent of the shared genetic risk and whether childhood ADHD (cADHD) influences the characteristics of the adult BP remain unclear. Our objectives were: (i) to test the ability of polygenic risk scores (PRS) derived from the latest PGC ADHD-GWAS (Demontis et al., 2019) to predict the presence of cADHD in BP patients; (ii) to examine the hypothesis that BP preceded by cADHD is a BP subtype with particular clinical traits and (iii) partially shares its molecular basis with ADHD.

Method: PRS derived from the ADHD-GWAS-2019 were tested in BP patients (N=942) assessed for cADHD with the Wender Utah Rating Scale and in controls from Romania and UK $(\mathrm{N}=1616)$.

Results: The ADHD-PRS differentiated BP cases with cADHD from controls. Proband sex and BP age-of-onset significantly influenced the discriminative power of the ADHD-PRS. The ADHD-PRS predicted the cADHD score only in males and in BP cases with early age-of-onset ( $\leq 21$ years). Bipolar patients with cADHD had a younger age-of-onset of mania/depression than patients without cADHD. The ADHD-PRS predicted the BP-affection status in the comparison of early-onset BP cases with controls suggesting a partial molecular overlap between early-onset BP and ADHD.

Limitations: Retrospective diagnosis of cADHD, small sample size.

Conclusions: The PRS-analysis indicated an acceptable predictive ability of the ADHD-SNP-set 2019 in independent BP samples. The best prediction of both cADHD and BP-affection status was found in the early-onset BP cases. The results may have impact on the individual disease monitoring.

Keywords: bipolar disorder; ADHD SNP set 2019; polygenic risk scores; prediction; age-of-onset; 


\section{Abstract (Version 2 for separate abstract submission) (249 words)}

Background: Although there is evidence of genetic correlation between bipolar disorder (BP) and ADHD, the extent of the shared genetic risk and whether childhood ADHD (cADHD) influences the characteristics of the adult BP remain unclear. Our objectives were: (i) to test the ability of polygenic risk scores (PRS) derived from the latest PGC ADHD-GWAS (Demontis et al., 2019) to predict the presence of cADHD in BP patients; (ii) to examine the hypothesis that BP preceded by $\mathrm{CADHD}$ is a BP subtype with particular clinical traits and (iii) partially shares its molecular basis with ADHD.

Method: PRS derived from the ADHD-GWAS-2019 were tested in BP patients (N=942) assessed for cADHD with WURS and in controls from Romania and UK $(\mathrm{N}=1616)$.

Results: The ADHD-PRS differentiated BP cases with cADHD from controls. Proband sex and BP age-of-onset significantly influenced the discriminative power of the ADHD-PRS. The ADHD-PRS predicted the cADHD score only in males and in BP cases with early age-of-onset ( $\leq 21$ years). Bipolar patients with cADHD had a younger age-of-onset of mania/depression than patients without cADHD. The ADHD-PRS predicted the BP-affection status in the comparison of early-onset BP cases with controls suggesting a partial molecular overlap between early-onset BP and ADHD.

Limitations: Retrospective diagnosis of cADHD, small sample size.

Conclusions: The PRS-analysis indicated an acceptable predictive ability of the ADHD-SNP-set 2019 in independent BP samples. The best prediction of both cADHD and BP-affection status was found in the early-onset BP cases. The results may have impact on the individual disease monitoring.

Keywords: bipolar disorder; ADHD SNP set 2019; polygenic risk scores; prediction; age-of-onset; 


\section{INTRODUCTION}

Family and high risk studies have observed an increased frequency of attention deficit/ hyperactivity disorder (ADHD) in the offspring of parents with major psychiatric disorders (Rieder et al, 1979; Decina et al, 1983; Gershon et al, 1985; Erlenmeyer-Kimling \& Cornblatt, 1987; GrigoroiuSerbanescu et al, 1989; 1991) suggesting that childhood ADHD (cADHD) may be an unspecified precursor of the adult psychoses.

A recent meta-analysis staging the developmental symptoms preceding full blown bipolar disorder (BP) (Raouna et al, 2018) showed that the childhood precursors of the disease mainly consist of non-mood disorders (anxiety disorders, ADHD, disruptive behavioural disorders). In a previous study on offspring aged 10-18 years of parents with BP type-1 (BP-I) we showed that ADHD was the most prevalent disorder in boys, while anxiety disorders and depressive spectrum disorders were the most prevalent disorders in girls compared with same sex offspring of healthy parents (GrigoroiuSerbanescu et al, 1989). A meta-analysis of family genetic studies of BP and ADHD patients (Faraone et al, 2012) investigated the comorbidity of ADHD and BP and reported a weighted prevalence of ADHD of $27.0 \%$ in the offspring of BP-I parents and $16.5 \%$ in the BP-I parents themselves compared with $9.6 \%$ in the offspring of controls and $4.5 \%$ in their parents. The weighted prevalence of BP-I was $6.8 \%$ in the offspring of ADHD parents and 5.1\% in the parents of ADHD probands compared with a weighted prevalence of BP-I of 3.5\% in the offspring of controls and 3.1\% in their parents. A metaanalysis of the most informative studies on offspring of parents with major psychoses (schizophrenia, BP, unipolar major depression) conducted over the last 50 years (Rasic et al, 2015) took into account the age dependent nature of ADHD prevalence and onset and subdivided the offspring by age into two groups ( $\leq 20$ years and $>20$ years). They reported that the rate of ADHD was $17 \%$ in offspring aged $\leq 20$ years of BP parents compared to $7 \%$ in offspring aged $>20$ years of BP parents. The ADHD rate 
of the total offspring group $(\mathrm{N}=1492)$ of BP parents was $14 \%$. All of the meta-analyses described above included part of the current Romanian BP-I sample.

\section{Conversely, long-term follow-up of children with ADHD at age 6 showed that $23 \%$ of them develop BP-I by the age 16 (Uchida et al, 2018).}

The figures from family genetic studies must be compared with prevalence figures in the general population. Polanczyk et al (2007) estimated the worldwide pooled prevalence of cADHD at 5.29\% in the general population aged $\leq 18$ years, while the mean prevalence in the adult population was estimated at $2.5 \%$ (Simon et al, 2009). In 2011 the prevalence of ADHD diagnosed according to DSM-IV criteria in the general European population aged 6-17 years was estimated at 5\% with a three times higher prevalence in boys than in girls (Wittchen et al, 2011). The same higher prevalence of ADHD in boys than in girls was observed in other general populations (Faraone et al, 2015).

The comorbidity between BP and ADHD varies according to BP subtype. As shown in a review by Skirrow et al. (2006) the comorbidity between BP-I and ADHD ranges between $22 \%$ and $61 \%$. In the study by Birmaher et al. (2006) the comorbidity between BP as global diagnosis and ADHD was $58.6 \%$, the comorbidity between BP-I and ADHD was $61.2 \%$, the comorbidity between BP-II and ADHD was 31.6\%. Birmaher et al. (2006) used K-SADS and DSM-IV criteria. In an epidemiologic survey conducted on a sample of 34,000 adult subjects retrospectively diagnosed (Bernardi, et al. 2012) the comorbidity between BP and ADHD was $33.56 \%$ (s.d. 29.37\%-38.03\%) in patients and 6.23\% in the general population.

Family studies (Faraone et al, 1997a; 1997b) advanced the hypothesis that BP with comorbid cADHD might be a BP subtype having particular clinical and possibly molecular characteristics. One 
of the characteristics would be an earlier age of onset (AO) of the disease, especially an earlier onset of mania (Faraone et al, 1997b). This view found support in some clinical studies. In a Swedish sample of 159 BP-I and BP-II cases (Ryden et al, 2009) retrospectively diagnosed with the Wender Utah Rating Scale all BP patients with cADHD had an earlier onset than BP patients without CADHD and the comobidity between BP and cADHD was $28.3 \%$. Nierenberg et al. (2005) found a 5 years younger AO and a higher rate of other comorbid psychiatric disorders in cases with lifetime ADHD than in cases without ADHD in a sample of $1000 \mathrm{BP}$ cases retrospectively diagnosed for comorbid ADHD. The prevalence of ADHD was $9.5 \%$ in the total BP sample, $14.7 \%$ in male patients and $5.8 \%$ in female patients.

The familial and high risk studies of BP and ADHD suggested that there may be partial sharing of the molecular genetic basis of the two disorders. The Brainstorm Consortium (2018) showed significant genetic correlations ( $\mathrm{rg}$ ) between $\mathrm{BP}$ and $\mathrm{ADHD}\left(\mathrm{rg}=0.26 ; \mathrm{P}=7 \times 10^{-06}\right)$.

Van Hulzen et al (2017), using an older ADHD GWAS, reported a stronger genetic correlation between $\mathrm{ADHD}$ and $\mathrm{BP}$ in the early-onset $\mathrm{BP}$ cases $(\mathrm{AO} \leq 21$ years $)\left(\mathrm{rg}=0.71 ; \mathrm{P}=4.0 \times 10^{-16}\right)$ than in the total BP sample $\left(0.64, \mathrm{P}=3.13 \times 10^{-14}\right)$. They also found a common significant region for $\mathrm{BP}$ and ADHD on chromosome 5 in the ADCY2 gene when combining early-onset BP cases with ADHD cases. This region had previously been reported to be associated with BP (Mühleisen et al, 2014). Both observational and molecular genetic studies suggest a greater overlap between ADHD and early onset BP. 
OBJECTIVES

Our first objective was to test the ability of PRS derived from the newest GWAS of ADHD (Demontis et al, 2019) to predict the presence of cADHD symptoms in BP subjects in independent samples different from the discovery samples.

The second objective was to test whether BP preceded by a history of cADHD may represent a subtype of BP with different clinical and possibly molecular characteristics, as suggested by previous studies. In particular, we explored the relationship between cADHD and BP-AO.

A third objective of our work was to explore the hypothesis of a partial molecular overlap between BP and ADHD in the limited context of the investigated ADHD-SNP set having in mind the developmental course of BP preceded by cADHD in a subgroup of BP patients.

\section{METHODS}

All patients and controls included in this study in Romania and the UK provided written informed consent. The study was approved by the ethical committees of the participating centres.

\subsection{Clinical diagnosis of the Romanian BP-I sample and controls}

470 BP-I patients [189 males (40.2\%); 281 females (58.2\%)] and 329 controls [143 males (43.5\%), 186 females (56.5\%)] with data that passed the genome-wide genotyping quality control were included in the study. All patients and controls were of Romanian descent. Genealogical information about parents and all four grandparents was obtained through direct interview of the subjects.

Unrelated BP-I patients were recruited from consecutive admissions in the Obregia Psychiatric Hospital of Bucharest. The patients were investigated with the Diagnostic Interview for Genetic Studies (DIGS) (Nurnberger et al, 1994). The diagnosis of BP-I was assigned according to DSM-IV criteria (APA, 1994) on the basis of both the DIGS and medical records. Patients were included in the sample 
if they had at least two documented hospitalized illness episodes (one manic/mixed and one depressive or two manic episodes) and no residual mood incongruent psychotic symptoms during remissions. This information was also confirmed by first degree relatives for $64 \%$ of the cases. The illness AO was defined as the age at which the proband first met DSM-IV criteria for a manic, mixed, or major depressive episode. The mean $\mathrm{AO}$ of $\mathrm{BP}$ in the Romanian sample was 26.36 years $(\mathrm{SD}=9.58)$. Population-based controls from Romania were screened with the DIGS for a lifetime history of any major affective or schizoaffective disorders, schizophrenia and any other psychosis, obsessivecompulsive disorder, eating disorders, alcohol/drug addiction. Unaffected controls were included in the study.

\subsection{Clinical diagnosis of the $U K \mathrm{BP}$ sample and controls}

The UK sample included 472 BP cases with cADHD data [165 males (34.95\%); 307 females (65.05\%)] and 1287 controls (34\% males; 66\% females). BP cases had been given a UK NHS clinical diagnosis of ICD-10 BP and then needed to fulfill Research Diagnostic Criteria (Spitzer et al, 1978) for BP with clinical data collected by the lifetime version of the Schizophrenia and Affective Disorder

Schedule (SADS-L) (Spitzer et al, 1977). Participants were also rated with the 90-item Operational Criteria Checklist (OPCRIT) (McGuffin et al, 1991). In the UK sample AO was defined as the age at which the proband first met criteria for a manic, mixed, or major depressive episode. The mean AO of $\mathrm{BP}$ in the UK sample was 25.8 years $(\mathrm{SD}=11.05)$. In the UK sample $67 \%$ of the cases were BP-I and $33 \%$ were BP-II.

The majority of the population-based UK controls were screened with the SADS-L for lifetime history of any major affective or schizoaffective disorders, schizophrenia and any other psychosis, 
obsessive-compulsive disorder, eating disorders, alcohol/drug addiction. Only unaffected controls were included in the study. 37.5\% (384) of the UK controls were blood donors obtained from ECACC (Public Health England, Porton Down, UK) and had not been screened for the presence of psychiatric disorders.

\subsection{Retrospective childhood ADHD symptom diagnosis in the Romanian and UK BP samples}

All 470 Romanian cases included in this study were directly interviewed either face to face or on phone with the 25 item version of the Wender Utah Rating Scale (WURS) (Ward et al, 1993) with regard to the presence of ADHD symptoms in their childhood. For many of the Romanian participants, clinical information about the presence of cADHD had already been obtained through items from the Kiddie-SADS clinical interview (1995) at their entry to the study.

The UK patients self-completed the WURS either on paper or through an online version. WURS data was available for 472 of the BP cases.

ADHD criteria were covered by the WURS items as follows: inattention (items 1, 6, 10,11 and 15); hyperactivity (items 5 and 29); impulsivity (items 7, 8, 9, 17, 21, 24, 26, 27, 28); emotional

instability (items 4, 12, 16, 20); significant functional impairment: (items $25,41,51,56,59$ ). A cut-off score of 46 differentiated the cases with cADHD from cases without cADHD as recommended by the WURS authors.

In both national samples the controls were not investigated for the presence of cADHD.

\section{Clinical similarities of the two national samples}

In clinical terms the two samples had similarities allowing the comparison of the molecular data. The mean ages of onset in the total samples and in subgroups of patients were very similar. The 
mean $\mathrm{AO}$ of $\mathrm{BP}$ in the total samples: $\mathrm{Romania}=26.36$ years $(\mathrm{SD}=9.58), \mathrm{UK}=25.8$ years $(\mathrm{SD}=11.05)$; the mean $\mathrm{AO}$ in cases with cADHD: Romania $=24.25$ years $(\mathrm{SD}=8.66), \mathrm{UK}=23.99(\mathrm{SD}=9.35)$; the mean $\mathrm{AO}$ in cases without $\mathrm{cADHD} \quad:$ Romania $=27.96(\mathrm{SD}=9.95), \mathrm{UK}=27.29(\mathrm{SD}=10.12)$. Both samples included more females than males and were diagnosed according to the same stringent DSMIV criteria and with the same scale for assessing cADHD (WURS), with the same cut-off point (46) for diagnosing ADHD. The assessment was made by clinicians in both samples.

\subsection{Age of onset group definition}

All BP samples (UK and Romanian) were subdivided by AO based on data from a prior admixture (commingling) analysis performed in the Romanian sample and two other independent samples (Grigoroiu-Serbanescu et al, 2014). This suggested dividing BP subjects into three AO groups: early onset defined with $\mathrm{AO} \leq 21$ years (49.1\% Romanian sample; $43 \%$ UK sample), intermediate onset with AO 22-34 years (38.5\% Romanian sample; 37\% UK sample), and late onset as AO>34 years (20.6\% Romanian sample; $20 \%$ UK sample). We then collapsed the intermediate and late onset groups due to small number of cases in the late onset group and termed this group "late-onset".

\subsection{Genotyping and imputation}

Genotyping of the Romanian sample. The BP-I cases and controls were genome-wide genotyped on Illumina Omni Express beadchips at the Institute of Human Genetics, Bonn, Germany. Genotyping QC was described elsewhere (Xiao et al, 2018). After QC 19 cases and 3 controls were excluded.

Genotyping of the UK sample. Subjects were genotyped using the Illumina PsychArray beadchip at the Broad Institute, MA, US. Stringent quality control was applied to the genotype 
information. Individuals were excluded on the basis of having incorrect gender assignments; excessive heterozygosity (more than 10 standard deviations above the mean); missing genotype data above $10 \%$ and evidence of relatedness. SNPs were excluded with a minor allele frequency $<0.5 \%$ and deviating substantially from the Hardy-Weinberg equilibrium $\left(\mathrm{P}<10^{-6}\right)$.

Imputation of the Romanian sample. Phasing and imputation of the quality controlled SNPset were performed with EAGLE (v2.3.5) (Loh et al, 2016) and Minimac3 (v2.0.1) (Das et al, 2016), based on 1000Genomes Project (Phase 3, v5). The typed variants were aligned to the human reference genome (GRCh37). Computational phasing was performed using Eagle (v2.3.5) with default parameters. Statistical genotype imputation was performed using Minimac3 (v2.0.1) with default parameters, and utilized publicly available reference haplotypes from the 1000 Genomes Project (Phase 3 , v5). Non-complementary SNPs with info/rsq $>0.8, \mathrm{MAF}>1 \%$ in the Romanian sample were chosen followed by LD clumping based on 1000 Genomes to remove SNPs with rsq $>0.1$ within $1 \mathrm{Mb}$ range. The pre-imputation SNP number was 528,877 and the post-imputation QC-ed number was 7,755,900 SNPs.

Principal component analysis (PCA) was performed with EIGENSTRAT for all of the 1000 Genomes Project subjects and for only the European 1000 Genomes subjects excluding Finnish samples. Non-complementary SNPs from the genotyped SNP set were merged with 1000 Genomes Project data (Phase 3, v5), followed by LD-clumping (500 SNP window, 100 SNP overlap, rsq $<0.05$ ). Outliers for heterozygosity and homozygosity were excluded (1 patient). Only SNPs with a MAF $>1 \%$, missing $<5 \%$, and HWE-P $>1 \mathrm{e}-6$ were included in the analysis. The lambda based on the Romanian sample was 1.011 and lambda1000 was 1.034 , thus indicating a relatively homogenous population with very little evidence of substructure.

Imputation of the UK sample. Imputation was performed using the Sanger Imputation server. Prior to upload of data genotypes were prepared as instructed and checks were performed using the 
HRC-1000G-check-bim tool Version 4.2.3 (Rayner, 2015). 393,270 SNPs with a MAF>0.01 were uploaded for imputation, applying the Haplotype Reference Consortium reference panel (release 1.1) (McCarthy et al, 2016). Pre-phasing was done with EAGLE v.2.3.3 and imputation was done with PBWT (Durbin, 2014). Post imputation QC excluded all SNPs with info $<0.9$, returning 10,326,133 SNPs.

\subsection{Computation of PRS in the Romanian and UK samples}

Discovery SNP set. For computing ADHD-PRS in the Romanian and UK samples we used summary ADHD GWAS data from the Psychiatric Genomics Consortium (Demontis et al, 2019) (https://www.med.unc.edu/pgc/results) as a discovery set (8,047,421 SNPs).

Target SNP set. Based on overlapping SNPs between the discovery set and the target sets at ten P-thresholds (PT) PRS-PTs were computed ranging from 0.01 (17,084 SNPs in the Romanian samples and 10,488 SNPs in the UK samples) to 0.50 (130,331 SNPs in the Romanian samples and 322,986 SNPs in the UK samples) using PRSice (Eusden et al, 2015).

\subsection{Statistical analysis}

The PRS scores were normalised using the Z-score method in order to make the data comparable between the two samples. The raw PRS-values were normally distributed at all PTs in both samples. Linear and logistic regressions of PRS were performed on the linear or dichotomous WURS score and on the BP case-control status using Rv.3.5.1 (R Core Team, 2018). ADHD-PRS analyses were performed independently in BP samples from Romania and from the UK. The results from both samples were then meta-analysed with the function "metagen" from the Meta package in R (Schwarzer, 2007). 
False discovery rate (FDR) correction (Benjamini \& Hochberg, 1995) for multiple testing was applied to the results obtained in the national samples and in the meta-analysis as implemented in the p.adjust function in R (R Core Team, 2018).

\section{RESULTS}

203 BP cases (43.19\%) in the Romanian sample and 162 BP cases (34.32\%) in the UK sample were diagnosed with cADHD.

\section{Objective 1}

In order to assess the ability of the ADHD-PRS based on the newest SNP set associated with ADHD to predict cADHD in independent samples we conducted several comparisons.

3.1. Differentiation of BP cases with cADHD from controls. The ADHD-PRS were able to nominally significantly differentiate BP cases with cADHD from controls at all PRS-PTs tested in the Romanian sample ( $\mathrm{P}=0.006$ to $\left.\mathrm{P}=0.036, \mathrm{R}^{2}=2 \%-1.1 \%\right)$ and in the meta-analysis $(\mathrm{P}=0.0012$ to $\mathrm{P}=0.021$ ) (Table 1), but not in the UK sample. After FDR correction, seven PRS-PTs remained significant in the metaanalysis (PRS_PT_0.02, PRS_PT_0.05 to PRS_PT_0.50) and four PRS_PTs (PT_0.10 to PT_0.50 in the Romanian sample).

3.2. Differentiation of BP cases with cADHD from BP cases without CADHD. We tested whether the ADHD-PRS could differentiate BP subjects with cADHD (N=365) from those without cADHD (N=577). In the meta-analysis all ten ADHD-PRS_PTs nominally differentiated the two groups ( $\mathrm{P}=0.011$ to $\mathrm{P}=0.027$ ), but after FDR correction only a trend to significance remained ( $\mathrm{P}=0.079$ to $\mathrm{P}=0.055$ ) (Table 2). The FDR correction of the results in the national samples did not show any significant difference between the two groups, although in the UK sample four PTs distinguished between BP subjects with and without cADHD at nominal level. 
3.3. The ADHD-PRS loading in BP cases with cADHD. The correlation of the ADHD_PRS_PTs with the WURS score in the total BP sample (Table 3) was positive and significant nominally ( $\mathrm{P}=0.0058$ to $\mathrm{P}=0.0008)$ and FDR-corrected $(\mathrm{P}=0.044$ to $\mathrm{P}=0.024)$ in the meta-analysis indicating that the higher the ADHD polygenic burden, the higher the WURS-cADHD score. In both national samples there was also a positive and significant correlation at the majority of the PRS_PTs at nominal level, but only one PRS_PT (PRS_PT_0.10) survived the FDR-correction in the Romanian sample.

3.4. Differentiation of total BP sample from controls. None of the ADHD-PRS was able to differentiate the total BP sample from controls in the meta-analysis (Table $\mathbf{S} \_\mathbf{1}$ ). There was however nominal evidence of differentiation in the Romanian sample for ADHD-PRS PTs between 0.1 and 0.5 ( $\mathrm{P}=0.026$ to $\mathrm{P}=0.035$ ), but this ADHD-PRS only explained $0.8 \%$ of the variance (Nagelkerke $\mathrm{R}^{2}$ ) and the differentiation disappeared after FDR-correction.

\subsection{Influence of proband sex on ADHD-PRS correlation with cADHD in BP patients. Given the} higher prevalence of cADHD amongst males in epidemiological studies, we conducted linear regressions of ADHD-PRS on WURS score in the BP samples with sex as covariate (Table S_2).

In the meta-analysis the influence of sex was significant both nominally ( $\mathrm{P}=0.0052$ to $\mathrm{P}=0.0004)$ and FDR-corrected at all PRS_PTs ( $\mathrm{P}=0.044$ to $\mathrm{P}=0.024)$ indicating a greater influence of the male sex (Table S_2). Nine PTs in the Romanian sample and seven PTs in the UK sample showed a nominally significant sex influence. These results explained up to $6.2 \%$ of the variance (PT_0.1, P=0.004) with significant sex effect $(t=-4.82, P=0.000)$ at all PTs in the Romanian sample and up to $1.3 \%$ of the variance (PT_0.01, $\mathrm{P}=0.014)$ with no significant evidence for a sex effect in the UK sample $(\mathrm{t}=-0.335$, $\mathrm{P}=0.738$ ). The sex effect had the same negative direction in both samples. Four PRS_PTs remained significant in the Romanian sample after FDR-correction. 
We then performed an analysis in separate sex groups. Table S_3a shows that the ADHD_PRS nominally significantly predicted the WURS cADHD in BP males in the combined sample at all PRS_PTs and at seven PRS_PTs in the Romanian sample indicating a better association of the ADHDPRS with the male sex. After FDR-correction the significance disappeared.

In the female subsample (Table S_3b) there was no significant prediction of the WURS score by the ADHD_PRS after FDR-correction, although a nominal significance was observed for three PRS_PTs (0.01-0.03) only in the combined sample.

\section{Objective 2}

3.6. Relationship between ADHD-PRS loading and age-of-onset of BP. The linear regressions of the ADHD-PRS on the linear measure of cADHD (linear WURS score) with the $\boldsymbol{B P}-\boldsymbol{A} \boldsymbol{O}$ as covariate (Table 4) showed a significant influence of BP-AO on the association of all ADHD-PRS_PTs with the cADHD score in BP patients at nominal level $(\mathrm{P}=0.0045$ to $\mathrm{P}=0.0009)$ and FDR corrected $\mathrm{P}$-level in the meta-analysis (corrected $\mathrm{P}=0.044$ to $\mathrm{P}=0.024$ ). In both national samples the majority of PRS_PTs were nominally significant, but after FDR-correction six PRS_PTs remained significant in the Romanian sample, while in the UK sample all PRS_PTs displayed only trends. The explained variance reached up to $15.7 \%$ in the Romanian sample (PT_0.10, nominal P=0.006) and up to $4.8 \%$ in the UK sample (PT_0.10, nominal $\mathrm{P}=0.012)$.

3.7. BP age of onset groups and ADHD-PRS loading. Based on the significant covariance effect of BP-AO on the association between patient cADHD score and ADHD-PRS, we investigated this association in $\mathrm{AO}$ groups to see if the association is uniform across the $\mathrm{AO}$ distribution or specific to an $\mathrm{AO}$ group that drives the association in the total sample.

Eight PTs in the Romanian sample and seven PTs in the UK sample predicted nominally significantly the cADHD score in the early-onset group, but only trends to significance remained after FDR- 
correction. Instead, in the combined sample the ADHD-PRS significantly predicted the cADHD score at all ADHD-PRS PTs both nominally ( $\mathrm{P}=0.015$ to $\mathrm{P}=0.0008$ ) and FDR-corrected ( $\mathrm{P}=0.032$ to $\mathrm{P}=0.007)$ in the early-onset BP group (Table 5). In the late-onset BP group, no ADHD PRS_PT predicted the cADHD score in either sample (Table $\left.\mathbf{S} \_4\right)$.

We investigated the mean BP-AO in cases with cADHD compared with cases without cADHD (Table S_5). In the Romanian sample the mean BP-AO was 24.25 years $(\mathrm{SD}=8.66)$ in cases with cADHD and 27.96 years $(\mathrm{SD}=9.95)$ in cases without $\mathrm{CADHD}(\mathrm{F}=17.89, \mathrm{P}<0.0001)$. In the UK sample the mean BP-AO was 23.99 years in cases with cADHD and 27.29 years in cases without cADHD $(\mathrm{F}=7.85, \mathrm{P}=0.0053)$.

In the Romanian sample we also observed the $\mathrm{AO}$ of mania and depression in cases with cADHD versus cases without $\mathrm{cADHD}$. Both the $\mathrm{AO}$ of mania $(\mathrm{P}<0.0001)$ and the $\mathrm{AO}$ of depression $(\mathrm{P}<0.005)$ were significantly younger in $\mathrm{BP}$ cases with $\mathrm{cADHD}$ as against cases without cADHD (Table S_5).

3.8. Independent association of ADHD-PRS with BP age-of-onset. Given the relationship between WURS score, BP-AO, and ADHD-PRS we tested whether AO was independently associated with ADHD-PRS (i.e. without taking into account the WURS score). There was a negative correlation (negative beta) between BP-AO and ADHD-PRS in all groups (Table S_6), suggesting that a higher ADHD-PRS loading was associated with a younger AO. But the corrected significance was shown only in the meta-analysis for PRS_PT_0.01. This means that only less frequent SNPs might influence the BP-AO.

\section{Objective 3}




\subsection{The hypothesis of an eventual partial molecular overlap between BP and ADHD in the context}

of the investigated ADHD-SNP set. In order to check this hypothesis we conducted logistic regressions where the predicted variable was the status "affected / not affected with BP" and the predictors were the ten ADHD-PRS-PTs. We first checked the prediction of the affection status in the comparison of BP cases without cADHD versus controls. The ADHD-PRS could not differentiate the BP patients without cADHD from controls in either sample (Table $\mathbf{S} \_7$ ), as they did in the comparison of the total BP samples with controls (Table S_1). But because the ADHD-PRS best predicted the cADHD in the early-onset BP cases, probably indicating an enriched and more homogeneous molecular basis of cADHD in this group, we compared the early-onset BP cases (including cases with and without cADHD) with the controls. Table 6 shows that eight PRS_PTs in the Romanian sample and seven PRS_PTs in the UK sample; and all ten PRS_PTs in the combined sample nominally significantly predict the status "affected - not affected with BP". After FDR correction of the meta-analysis nine PRS_PTs remained significant. This finding points to a partial molecular overlap between early-onset $\mathrm{BP}$ and ADHD.

Because the proband sex had a significant influence on the prediction of cADHD score in the total BP sample (Table S_2) and it was hypothesized that "there may be sex differences in the genetic overlap between ADHD and comorbid features" (Du Rietz et al, 2018), we investigated the sex effect on the overlap between early-onset BP and ADHD through the differentiation between early-onset group and controls based on ADHD-PRS with the covariate sex (Table S_8). The meta-analysis shows that nine of ten PTs are nominally significant and six PTs (PT_0.05 to PT_0.50) remain significant after FDRcorrection.

\section{DISCUSSION}

To the best of our knowledge, we are among the first researchers to use the newest PGC ADHDSNP set to investigate ADHD as a trait and developmental precursor of BP. We set out to investigate 
the ability of the ADHD-PRS to predict the presence of cADHD in BP subjects and to test whether BP preceded by a history of cADHD presents particular clinical characteristics, as suggested by previous studies (Faraone et al, 1997a; 1997b, Sachs et al. 2000).

\subsection{Predictive power of ADHD-PRS}

In the total BP sample all ADHD_PRS_PTs based on the PGC-SNP set demonstrated a significant positive correlation with the linear WURS score as measure of cADHD indicating that the higher the ADHD polygenic burden, the higher the WURS-cADHD score. The ADHD-PRS significantly differentiated the BP cases with cADHD from controls at seven PRS-PTs. However, in the comparison of $\mathrm{BP}$ cases with $\mathrm{cADHD}$ with BP cases without $\boldsymbol{c A D H D}$ only a trend to significance was observed after FDR-correction of the results, although at nominal level the differentiation was significant. Likewise, the ADHD_PRS did not differentiate the total BP samples from controls. This replicates the findings of Du Rietz et al (2018), who used the same ADHD discovery set and could not distinguish between BP patients and controls using ADHD-PRS to predict the status "affected -not affected with BP" in a large UK biobank sample. These findings might be due to the common character of the SNPs included in PRS, which means that many individuals have PRS-loading close to the population mean and consequently, have the general "population life-time disease risk" (Lewis \& Vassos, 2017). Another explanation resides in the fact that the PRS consists of SNPs associated with ADHD, but the total sample of BP cases includes subjects with and without cADHD. Similar to our study, the controls of Du Rietz et al. study (2018) were not investigated for the presence of cADHD.

The predictive power of the ADHD-PRS for CADHD was influenced by the proband sex in the BP samples. A nominally significant prediction of cADHD was found in the Romanian and the combined BP male sample at all PRS_PTs, while no significant prediction was observed in female patients in either sample when the analysis was performed in separate sex groups. This result is in line with the double ADHD-SNP heritability reported for male subjects $\left(\left(\mathrm{h}^{2}=0.247\right)\right.$ compared to female 
subjects $\left(\mathrm{h}^{2}=0.123\right)$ (Martin et al, 2018) and with the observation that "there may be sex differences in the genetic overlap between ADHD and comorbid features" (Du Rietz et al, 2018), although the FDRcorrection vanished the sex significance in our samples. These studies used the same ADHD-SNP set used by us and explained the sex difference in SNP heritability through heterogeneous effects of rare or non-autosomal variants, although sex heterogeneity of common variants could not be excluded. This heterogeneity seems to have worked also in our samples, since the greatest sex effect was more visible in the Romanian and the combined samples.

\subsection{Clinical characteristics of BP preceded by cADHD and partial overlap between BP and ADHD}

The AO of BP significantly influenced the predictive power of ADHD-PRS for cADHD both in the national samples and in the combined sample at all PRS_PTs. When the sample was devided into an early-onset and a late-onset group we found that the WURS score as measure of cADHD was predicted by PRS only in early-onset $\mathrm{BP}$ cases ( $\mathrm{AO} \leq 21$ years), but not in late-onset $\mathrm{BP}$ cases. This finding and the fact that $43.19 \%$ cases in the Romanian sample and $34.32 \%$ cases in the UK sample had cADHD support the clinical observation that cADHD may be a non-specific behavioral precursor of future adult BP (Ryden et al., 2009; Uchida et al., 2018; Raouna et al., 2018) and specifically of early-onset BP (Faraone et al, 1997b; Sachs et al, 2000; Nierenberg et al, 2005).

The AO distribution in both the RO and UK sample was in agreement with the AO distribution of $\mathrm{BP}$ in an worldwide sample (Merikangas et al, 2011) according to whom 50\% of BP cases have onset by age 25. It is well known that the AO distribution in BP is skewed to the left. So the best prediction of cADHD in BP cases with early-onset was not artificially driven by a biased AO distribution.

As a clinical characteristic of the BP preceeded by cADHD we found a significantly younger $\mathrm{BP}-\mathrm{AO}$ in cases with cADHD than in cases without cADHD in both national samples. In the Romanian sample, where data on $\mathrm{AO}$ of mania and depression were available, we also found significantly younger 
AO of mania and depression in patients with cADHD. Thus, we could confirm the observation according to which BP comorbid with cADHD has an earlier onset.

For testing the hypothesis of a partial molecular overlap between BP and ADHD suggested by family studies and genetic correlations in large GWAS studies, we used the paradigm of the dependent variable "affected - not affected with BP" predicted by the ten ADHD-PRS-PTs in several group comparisons. Although we could not differentiate the BP patients without cADHD from controls using this model, we could nevertheless significantly distinguish between the total early-onset BP group (including cases with and without cADHD) and controls in the meta-analysis of the Romanian and UK sample. This finding is suggestive for the partial molecular overlap of ADHD and BP with early-onset and replicates the study of Van Hulzen et al (2017) based on an older ADHD-GWAS.

\subsection{Study limitations}

The main limitation of our study was that cADHD was retrospectively assessed in the BP patients using the WURS and as such the data could be influenced by recall bias which, in turn, could be influenced by the severity of BP. Information provided by close relatives was available for many Romanian patients, but this information was also retrospectively obtained. We used the recommended cut-off of 46 points on the 25 item WURS for diagnosing cADHD and this score is reached through the inclusion of items which are not very specific for ADHD, although they are related (e.g. items for emotional instability, items more specific for conduct disorders and intellectual performance). Emotional instability is not included among the core criteria of ADHD, although this symptom is seen in ADHD children in clinical practice. Emotional instability overlaps with symptoms of BP and, in different degrees, it is a trait common to several disorders and personality structures in the general population, which might have contributed to the lack of differentiation between the total BP sample and controls based on ADHD-PRS. As stated by Ward et al. (1993) the cut-off 46 or higher on WURS 
correctly identifies $86 \%$ of cases with cADHD, while a cutoff score of 36 or higher correctly identifies $96 \%$ of the adults with cADHD (sensitivity $=96 \%$ ), but this threshold is used less frequently.

The diagnostic sensitivity and specificity of the WURS varies from sample to sample depending on the items included in the cut-off and on the administration way (clinical interview or self-rating) with poorest results in self-rating (Suhr et al, 2009). In different adult samples retrospectively diagnosed with ADHD WURS had a sensitivity up to $96 \%$ and specificity from 70\% (Kouros et al. 2018), 76\% (Retz-Junginger et al. 2003) to $90.8 \%$ (Rodríguez-Jiménez et al., 2001). Having in mind that the specificity of a diagnostic tool is defined as the capacity to exclude those subjects who do not possess a certain trait, we appreciate that the WURS diagnostic properties did not significantly affect the percentage of BP patients with comorbid cADHD in our samples.

Although the reported rates of comorbidity in our samples might seem high and might suggest a bias introduced by the psychometric properties of WURS, in fact they are in the range of previous comorbidity studies between BP and ADHD mentioned in the introduction. Since the comorbidity varies according to BP-subtype and is higher between BP-I and ADHD, it is reasonable to find a higher comorbidity in the Romanian BP-I sample than in the UK sample consisting of 67\% BP-I cases and $33 \%$ BP-II cases.

Another limitation of our study resides in the different number of SNPs genotyped and imputed in the national samples. This may have influenced the comparability of the results in the two samples, although the PRS were Z-normalized for comparison and meta-analysis. An additional limitation of the study is derived from the current status of the biological basis of PRS technique that involves the deficiencies mentioned above.

\subsection{Conclusions}


Our study is amongst the first to investigate the predictive power of an ADHD-PRS set based on the most recent PGC ADHD-GWAS in bipolar disorder. We were able to confirm the discriminative power of the ADHD-SNPs by showing that they can differentiate between BP patients with cADHD and controls and tend to differentiate also between BP patients with cADHD and BP patients without cADHD if stringent correction for multiple testing is undertaken. Proband sex and BP-AO influenced the predictive power of the ADHD-PRS. The most robust finding of our study was the relationship between ADHD-PRS loading and BP-AO with significant association between ADHD-PRS and earlyonset $\mathrm{BP}$ suggesting that ADHD-PRS loading has a modifying impact on BP-AO. This result is also in agreement with findings of previous molecular studies of BP showing differences in the association of several candidate genes with BP as a function of the disease onset (Jamain et al, 2014; GrigoroiuSerbanescu et al, 2015). Our findings were also suggestive for the partial molecular overlap of ADHD and BP with early-onset. However, the ADHD-PRS only explained a small proportion of the variance that influences cADHD symptoms preceding BP.

Given the individual variability of treatment response and outcome of BP the use of an ADHDPRS in BP might contribute to personalized disease monitoring by stratification of the individual risk and tailoring the clinical treatment decisions according to the individual biological backgorund. Probably the greatest predictive benefit might be in cases situated at the extremes of the PRS distributions (Lewis \& Vassos, 2017). However, it remains to be determined whether there is an increased benefit of the ADHD-PRS over and above clinical investigation of AO and of childhood ADHD. Due to the sample size in this study, the findings that we report remain suggestive and require independent replication. 


\section{References}

Benjamini Y, Hochberg Y, 1995. Controlling the false discovery rate: a practical and powerful approach to multiple testing. Journal of the Royal Statistical Society. Series B 57, 289-300.

Bernardi S, Faraone SV, Cortese S, Kerridge BT, Pallanti S, Wang S, Blanco C, 2012. The lifetime impact of attention deficit hyperactivity disorder: results from the National Epidemiologic Survey on Alcohol and Related Conditions (NESARC), Psychological Medicine, 42, 875-887.

Birmaher, et al., Clinical course of children and adolescents with bipolar spectrum disorders. Archives of General Psychiatry, 2006, 63, 175-183)

The Brainstorm Consortium, 2018. Analysis of shared heritability in common disorders of the brain. Science 360: 6395, eaap8757.

Das S, Forer L, Schönherr S, Sidore C, Locke AE, Kwong A, et al, 2016. Next-generation genotype imputation service and methods. Nat Genet. 48, 1284-1287.

Decina P, Kestenbaum CJ, Farber S, Kron L, Gargan M, Sackeim HA, et al, 1983. Clinical and psychological assessment of children of bipolar probands. Am J Psychiatry 140, 548-553.

Demontis D, Walters RK, Martin J, Mattheisen M, Als TD, Agerbo E, et al, 2019. Discovery of the first genome-wide significant risk loci for ADHD. Nat Genet. 51, 63-75.

Du Rietz E, Jonathan Coleman, Kylie Glanville, Shing Wan Choi, Paul F. O’Reilly, et al, 2018. Association of polygenic risk for attention-deficit/hyperactivity disorder with co-occurring traits and disorders. Biol Psychiatry: Cognitive Neuroscience and Neuroimaging 3, 635-643.

Durbin R, 2014. Efficient haplotype matching and storage using the positional Burrows-Wheeler transform (PBWT). Bioinformatics 30, 1266-1272. 
Erlenmeyer-Kimling L, Cornblatt B, 1987. High-risk research in schizophrenia: a summary of what has been learned. J Psychiatr Res 21, 401-411.

Euesden J, Lewis CM, O'Reilly PF, 2015. PRSice: Polygenic Risk Score software. Bioinformatics 31, 1466-1468.

Faraone SV, Biederman J, Mennin D, Wozniak J, Spencer T, 1997a. Attention-deficit hyperactivity disorder with bipolar disorder: a familial subtype ? J Am Acad Child Adolesc Psychiatry 36, 137887.

Faraone SV, Biederman J, Wozniak J, Mundy E, Mennin D, O’Donnell D, 1997b. Is comorbidity with ADHD a marker for juvenile onset mania? J AmAcad Child Adolesc Psychiatry 36, 1046 -1055.

Faraone SV, Biederman J, Wozniak J, 2012. Examining the comorbidity between attention deficit hyperactivity disorder and bipolar disorder: a meta-analysis of family-genetic studies. Am J Psychiatry 169, 1256-1266.

Faraone SV, Asherson Ph, Banaschewski T, Biederman J, Buitelaar JK, Ramos-Quiroga JA, et al, 2015. Attention-deficit/hyperactivity disorder. Primer. Nature Reviews. Disease Primers 1: Article no: 15020.

Gershon ES, McKnew D, Cytryn L, Hamovit J, Schreiber J, Hibbs E, et al, 1985. Diagnoses in schoolage children of bipolar affective disorder patients and normal controls. J Affect Disord 8, 283-291.

Grigoroiu-Serbanescu M, Christodorescu D, Jipescu I, Totoescu A, Marinescu E, Ardelean V, 1989. Psychopathology in children aged 10-17 of bipolar parents: psychopathology rate and correlates of the severity of the psychopathology. J Affect Disord. 16, 167-179. 
Grigoroiu-Serbanescu M, Christodorescu D, Măgureanu S, Jipescu I, Totoescu A, Marinescu E, Popa S, 1991. Adolescent offspring of endogenous unipolar depressive parents and of normal parents. J Affect Disord 21, 185-198.

Grigoroiu-Serbanescu M, Rietschel M, Hauser J, Czerski PM, Herms S, Sun X, Wickramaratne P, Elston RC. 2014. Commingling analysis of age-of-onset in bipolar I disorder and the morbid risk for major psychoses in first degree relatives of bipolar I probands. J Affect Disord. 168, 197-204.

Grigoroiu-Serbanescu M, Diaconu CC, Heilman-Heimbach S, Neagu AI, Becker T, 2015. Association of age-of-onset groups with GWAS significant schizophrenia and bipolar disorder loci in Romanian bipolar I patients. Psychiatry Research 230, 860-863.

Jamain S, Cichon S, Etain B, Mühleisen TW, Georgi A, Zidane N, et al, 2014. Common and rare variant analysis in early-onset bipolar disorder vulnerability. PLoS One 9, e104326. doi: 10.1371/journal.pone.0104326. eCollection 2014.

Kiddie-Sads-Present and Lifetime Version (K-SADS-PL). Version 1.0 of October 1996. http: Ilwww.wpic.pitt.edulksads.

Kouros I, Hörberg N, Ekselius L, Ramklint M, 2018. Wender Utah Rating Scale-25 (WURS-25): psychometric properties and diagnostic accuracy of the Swedish translation. Ups J Med Sci 123, 230-236.

Lewis CM, Vassos E, 2017. Prospects for using risk scores in polygenic medicine. Genome Med. 9, 96-98.

Loh P R, Danecek P, Palamara P F, Fuchsberger C, Reshef Y A, Finucane H K, et al , 2016. Referencebased phasing using the Haplotype Reference Consortium panel. Nat Genet. 48, 1443-1448. 
Martin J, Walters RK, Demontis D, Mattheisen M, Lee SH, Robinson E, et al, 2018. A Genetic Investigation of Sex Bias in the Prevalence of Attention-Deficit/Hyperactivity Disorder. Biol Psychiatry , 83, 1044-1053.

McCarthy S, Das S, Kretzschmar W, Delaneau O, Wood A R, Teumer A, et al, 2016. A reference panel of 64,976 haplotypes for genotype imputation. Nat Genet. 48, 1279-1283.

McGuffin P, Farmer A, Harvey I, 1991. A polydiagnostic application of operational criteria in studies of psychotic illness. Development and reliability of the OPCRIT system. Arch Gen Psychiatry 48, 764-770.

Merikangas KR, Jin R, He JP, Kessler RC, Lee S, Sampson NA et al. Prevalence and correlates of bipolar spectrum disorder in the world mental health survey initiative. Arch Gen Psychiatry. 2011; $68(3): 241-251$.

Mühleisen TW, Leber M, Schulze TG, Strohmaier J, Degenhardt F, Treutlein J, et al, 2014. Genomewide association study reveals two new risk loci for bipolar disorder. Nat Comm. 2014; 5:3339.

Nierenberg AA, Miyahara S, Spencer T, Wisniewski SR, Otto MW, Simon N, et al, 2005. Clinical and diagnostic implications of lifetime attention-deficit/hyperactivity disorder comorbidity in adults with bipolar disorder: Data from the first 1000 STEP-BD participants. Biol Psychiatry 57, 1467 1473.

Nurnberger JI, Blehar MC, Kaufmann CA, York-Cooler C, Simpson SG, Harkavy-Friedman J, et al, 1994. Diagnostic Interview for Genetic Studies. Rationale, Unique Features, and Training. Arch Gen Psychiatry 51, 849-859.

Polanczyk G, de Lima MS, Horta BL, Biederman J, Rohde LA, 2007. The worldwide prevalence of ADHD: a systematic review and metaregression analysis. Am J Psychiatry , 164, 942-948. 
R Core Team, 2018. A language and environment for statistical computing. R Foundation for Statistical Computing, Vienna, Austria. https://www.R-project.org/.

Raouna A, Osam CS, MacBeth A, 2018. Clinical staging model in offspring of parents with bipolar disorder: a systematic review. Bipolar Disord 20, 313-333.

Rasic D, Hajek T, Alda M, Uher R. (2014) Risk of mental illness in offspring of parents with schizophrenia, bipolar disorder, and major depressive disorder: a meta-analysis of family high-risk studies. Schizophr Bull 40, 28-38.

Rayner W, 2015. HRC-1000G-check-bim. Retrieved August 15, 2018, from http://www.well.ox.ac.uk/ wrayner/tools/

Rieder RO, Nichols PL, 1979. Offspring of schizophrenics. III. Hyperactivity and neurological soft signs. Arch Gen Psychiatry 36, 665-674.

Ryden E, Thase ME, Straht D, Aberg-Wistedt A, Bejerot S, Landen M, 2009. A history of childhood attention-deficit hyperactivity disorder (ADHD) impacts clinical outcome in adult bipolar patients regardless of current ADHD. Acta Psychiatr Scand . 120, 239-246.

Sachs GS, Baldassano CF, Truman CJ, Guille C, 2000. Comorbidity of attention deficit hyperactivity disorder with early- and late-onset bipolar disorder. Am J Psychiatry 157, 466-468.

Simon V, Czobor P, Balint S, Meszaros A, Bitter I, 2009. Prevalence and correlates of adult attentiondeficit hyperactivity disorder: meta-analysis. Br J Psychiatr 194: 204-211.

Skirrow C, Hosang GM, Farmer AE, Asherson P, 2006. An update on the debated association between ADHD and bipolar disorder across the lifespan. J Affect Disord, 2006, 141, 143-159. 
Spitzer RL, Endicott J, Robins E, 1978. Research diagnostic criteria: rationale and reliability. Arch Gen Psychiatry 35, 773-782.

Spitzer RL, Endicott J, 1977. The Schedule for Affective Disorder and Schizophrenia, Lifetime Version. New York, New York State Psychiatric Institute.

Schwarzer, G, 2007. Meta: An R package for meta-analysis. $R$ News 7, 40-45.

Suhr J, Zimak E, Buelow M, Fox L, 2009. Self-reported childhood attention-deficit/ hyperactivity disorder symptoms are not specific to the disorder. Comprehensive Psychiatry 50, 269-275.

Uchida M, Spencer TJ, Faraone SV, 2018. Adult Outcome of ADHD: An Overview of Results From the MGH Longitudinal Family Studies of Pediatrically and Psychiatrically Referred Youth With and Without ADHD of Both Sexes. J Atten Disord. 22, 523-534.

Van Hulzen KJE, Scholz CJ, Franke B, Ripke S, Klein M, McQuillin A, et al. 2017. Genetic overlap between attention-deficit/hyperactivity disorder and bipolar disorder: Evidence from genome-wide association study meta-analysis. Biol Psychiatry 82, 634-641.

Ward MF, Wender PH, Reimherr FW, 1993. The Wender Utah Rating Scale: an aid in the retrospective diagnosis of childhood attention deficit hyperactivity disorder. Am J Psychiatry 150, 885-890.

Wittchen HU, Jacobi F, Rehm J, Gustavsson A, Svensson M, Jönsson B et al, 2011. The size and burden of mental disorders and other disorders of the brain in Europe 2010. European Neuropsychopharmacology 21, 655-679.

Xiao X, Zhang C, Grigoroiu-Serbanescu M, Wang L, Li L, Zhou D, et al. 2018. The cAMP responsive element-binding (CREB)-1 gene increases risk of major psychiatric disorders. Mol Psychiatry 23, 1957-1967. 
1000 Genomes Project Consortium, 2010. A map of human genome variation from population-scale sequencing. Nature 467, 1061-1073. 


\section{Funding}

The recruitment, diagnosis and genotyping of the Romanian samples of patients and controls were funded by the UEFISCDI, Bucharest, Romania through the grant number 89/2012 to Dr. Maria Grigoroiu-Serbanescu. The UCL clinical and control samples were collected with support from Bipolar UK (formerly the UK Manic Depression Fellowship), the Neuroscience Research Charitable Trust, the Central London NHS (National Health Service) Blood Transfusion Service, the Camden and Islington NHS Foundation Trust, a research lectureship from the Priory Hospitals and the National Institutes for Health Research (NIHR) Mental Health Research Network (MHRN) and the Primary Care Research Network (PCRN). Genotyping of the UK samples was funded by the Stanley Center for Psychiatric Research at the Broad Institute. Genetic analysis was supported by UK MRC project grants G9623693N, G0500791, G0701007, G0801038, and G1000708. Drs McQuillin and Bass are supported by the UCLH NIHR BRC. 
Supplementary material: Supplementary tables S_1_8 
Table 1

ADHD_PRS in bipolar patients with cADHD and healthy controls (Logistic regression with dependent variable affected with cADHD - not affected with cADHD)

\begin{tabular}{|c|c|c|c|c|c|c|c|c|c|c|c|c|c|c|c|c|c|}
\hline \multirow{2}{*}{ PRS_PT } & \multicolumn{5}{|c|}{ Romanian Data } & \multicolumn{6}{|c|}{ UK Data } & \multicolumn{6}{|c|}{ Fixed Effect Meta-Analysis } \\
\hline & OR & SE & $95 \% \mathrm{CI}$ & $\mathbf{R 2}$ & $\mathbf{P}$ & FDR_P & OR & SE & $95 \% \mathrm{CI}$ & $\mathbf{R 2}$ & $\mathbf{P}$ & FDR_P & OR & $95 \% \mathrm{CI}$ & $\mathbf{Z}$ & $\mathbf{P}$ & FDR_P \\
\hline 0.01 & 0.2 & 0.09 & $(0.01-0.38)$ & 0.01 & 0.036 & 0.091 & 0.1 & 0.08 & $(-0.07-0.26)$ & 0.002 & 0.24 & 0.297 & 0.14 & $(0.02-0.26)$ & 2.26 & 0.0241 & 0.075 \\
\hline 0.02 & 0.22 & 0.09 & $(0.04-0.4)$ & 0.01 & 0.02 & 0.068 & 0.12 & 0.08 & $(-0.04-0.28)$ & 0.003 & 0.15 & 0.209 & 0.16 & $(0.04-0.28)$ & 2.61 & 0.0091 & 0.049 \\
\hline 0.03 & 0.22 & 0.09 & $(0.04-0.4)$ & 0.01 & 0.02 & 0.068 & 0.1 & 0.08 & $(-0.06-0.27)$ & 0.002 & 0.2 & 0.257 & 0.15 & $(0.03-0.27)$ & 2.5 & 0.0124 & 0.057 \\
\hline 0.04 & 0.21 & 0.09 & $(0.03-0.39)$ & 0.01 & 0.024 & 0.075 & 0.11 & 0.08 & $(-0.05-0.28)$ & 0.003 & 0.16 & 0.217 & 0.16 & $(0.04-0.28)$ & 2.53 & 0.0114 & 0.057 \\
\hline 0.05 & 0.23 & 0.09 & $(0.05-0.41)$ & 0.02 & 0.015 & 0.058 & 0.12 & 0.08 & $(-0.04-0.28)$ & 0.003 & 0.14 & 0.191 & 0.17 & $(0.05-0.29)$ & 2.73 & 0.0063 & 0.044 \\
\hline 0.1 & 0.25 & 0.09 & $(0.07-0.43)$ & 0.02 & 0.007 & 0.044 & 0.15 & 0.08 & $(-0.02-0.31)$ & 0.004 & 0.08 & 0.122 & 0.19 & $(0.07-0.31)$ & 3.11 & 0.0019 & 0.027 \\
\hline 0.2 & 0.25 & 0.09 & $(0.07-0.43)$ & 0.02 & 0.007 & 0.044 & 0.14 & 0.08 & $(-0.02-0.3)$ & 0.004 & 0.09 & 0.139 & 0.19 & $(0.07-0.31)$ & 3.07 & 0.0021 & 0.029 \\
\hline 0.3 & 0.25 & 0.09 & $(0.07-0.43)$ & 0.02 & 0.007 & 0.044 & 0.14 & 0.08 & $(-0.03-0.3)$ & 0.004 & 0.1 & 0.151 & 0.19 & $(0.06-0.31)$ & 3.01 & 0.0026 & 0.034 \\
\hline 0.4 & 0.25 & 0.09 & $(0.07-0.43)$ & 0.02 & 0.006 & 0.044 & 0.16 & 0.08 & $(-0.01-0.32)$ & 0.005 & 0.06 & 0.109 & 0.2 & $(0.08-0.32)$ & 3.23 & 0.0012 & 0.024 \\
\hline 0.5 & 0.25 & 0.09 & $(0.07-0.43)$ & 0.02 & 0.007 & 0.044 & 0.16 & 0.08 & $(-0.01-0.32)$ & 0.005 & 0.06 & 0.109 & 0.2 & $(0.08-0.32)$ & 3.23 & 0.0012 & 0.024 \\
\hline
\end{tabular}

PRS_PT $=$ P value threshold for ADHD polygenic risk score $\mathrm{R}^{2}=$ Nagelkerke $\mathrm{R}^{2}$ (percentage of explained variance); FDR_P $=$ false rate discovery corrected $\mathrm{P}$ for multiple testing $; 95 \% \mathrm{CI}=95 \%$ confidence intervals. 
Table 2.

Differentiation of bipolar patients with cADHD (WURS $\geq 46$ ) from bipolar patients without cADHD (WURS < 46) by the ADHD_PRS (Logistic regression with cADHD as dichotomous dependent variable).

\begin{tabular}{|c|c|c|c|c|c|c|c|c|c|c|c|c|c|c|c|c|c|}
\hline \multirow{2}{*}{ PRS_PT } & \multicolumn{6}{|c|}{ Romanian Data } & \multicolumn{6}{|c|}{ UK Data } & \multicolumn{5}{|c|}{ Fixed Effect Meta-Analysis } \\
\hline & OR & SE & $95 \% \mathrm{CI}$ & $\mathbf{R}^{2}$ & $\mathbf{P}$ & FDR_P & OR & SE & $95 \% \mathrm{CI}$ & $\mathbf{R}^{2}$ & $\mathbf{P}$ & FDR_P & OR & $95 \% \mathrm{CI}$ & $\mathbf{Z}$ & $\mathbf{P}$ & FDR_P \\
\hline 0.01 & 0.11 & 0.09 & $(-0.08-0.29)$ & 0.004 & 0.25 & 0.297 & 0.24 & 0.10 & $(0.05-0.44)$ & 0.018 & 0.015 & 0.058 & 0.17 & $(0.04-0.31)$ & 2.52 & 0.012 & 0.057 \\
\hline 0.02 & 0.11 & 0.09 & $(-0.07-0.29)$ & 0.004 & 0.25 & 0.297 & 0.23 & 0.10 & $(0.03-0.42)$ & 0.016 & 0.022 & 0.073 & 0.16 & $(0.03-0.3)$ & 2.42 & 0.015 & 0.058 \\
\hline $\mathbf{0 . 0 3}$ & 0.12 & 0.09 & $(-0.06-0.3)$ & 0.005 & 0.2 & 0.250 & 0.19 & 0.10 & $(0-0.39)$ & 0.011 & 0.049 & 0.103 & 0.15 & $(0.02-0.29)$ & 2.29 & 0.022 & 0.072 \\
\hline 0.04 & 0.12 & 0.09 & $(-0.07-0.3)$ & 0.005 & 0.2 & 0.257 & 0.18 & 0.10 & $(-0.01-0.38)$ & 0.01 & 0.062 & 0.109 & 0.15 & $(0.02-0.28)$ & 2.21 & 0.027 & 0.079 \\
\hline 0.05 & 0.14 & 0.09 & $(-0.05-0.32)$ & 0.006 & 0.15 & 0.200 & 0.18 & 0.10 & $(-0.01-0.37)$ & 0.01 & 0.063 & 0.109 & 0.16 & $(0.03-0.29)$ & 2.34 & 0.02 & 0.068 \\
\hline 0.1 & 0.16 & 0.09 & $(-0.03-0.34)$ & 0.008 & 0.1 & 0.151 & 0.2 & 0.1 & $(0-0.39)$ & 0.012 & 0.046 & 0.101 & 0.18 & $(0.04-0.31)$ & 2.55 & 0.011 & 0.055 \\
\hline 0.2 & 0.15 & 0.09 & $(-0.04-0.34)$ & 0.007 & 0.12 & 0.168 & 0.17 & 0.10 & $(-0.02-0.37)$ & 0.009 & 0.085 & 0.132 & 0.16 & $(0.03-0.3)$ & 2.32 & 0.02 & 0.069 \\
\hline 0.3 & 0.16 & 0.09 & $(-0.03-0.35)$ & 0.008 & 0.1 & 0.145 & 0.16 & 0.10 & $(-0.04-0.35)$ & 0.007 & 0.12 & 0.168 & 0.16 & $(0.02-0.29)$ & 2.27 & 0.023 & 0.074 \\
\hline 0.4 & 0.16 & 0.09 & $(-0.03-0.35)$ & 0.008 & 0.1 & 0.145 & 0.18 & 0.1 & $(-0.02-0.38)$ & 0.01 & 0.072 & 0.118 & 0.17 & $(0.03-0.3)$ & 2.43 & 0.015 & 0.058 \\
\hline 0.5 & 0.15 & 0.09 & $(-0.03-0.34)$ & 0.007 & 0.11 & 0.155 & 0.19 & 0.1 & $(-0.01-0.38)$ & 0.01 & 0.063 & 0.109 & 0.17 & $(0.03-0.31)$ & 2.45 & 0.015 & 0.058 \\
\hline
\end{tabular}

PRS_PT $=\mathrm{P}$ value threshold for ADHD polygenic risk score; $\mathrm{R}^{2}=$ Nagelkerke $\mathrm{R}^{2}$ (percentage of explained variance); FDR_P $=$ false rate discovery corrected P for multiple testing 
Table 3.

Correlation of ADHD PRS with linear WURS score in bipolar patients (total samples)

\begin{tabular}{|c|c|c|c|c|c|c|c|c|c|c|c|c|c|c|c|c|c|}
\hline \multirow{2}{*}{ PRS_PT } & \multicolumn{5}{|c|}{ Romanian Data } & \multirow[b]{2}{*}{ FDR_P } & \multicolumn{5}{|c|}{ UK Data } & \multirow[b]{2}{*}{ FDR_P } & \multicolumn{5}{|c|}{ Fixed Effect Meta-Analysis } \\
\hline & Beta & SE & $95 \% \mathrm{CI}$ & $\overline{\mathbf{R}^{2}}$ & $\mathbf{P}$ & & Beta & SE & $95 \% \mathrm{CI}$ & $\mathbf{R}^{2}$ & $\mathbf{P}$ & & Beta & $95 \% \mathrm{CI}$ & $\mathbf{Z}$ & $\mathbf{P}$ & FDR_P \\
\hline 0.01 & 1.04 & 0.58 & $(-0.1-2.17)$ & 0.007 & 0.074 & 0.119 & 2.48 & 1.02 & $(0.49-4.47)$ & 0.012 & 0.02 & 0.058 & 1.39 & $(0.4-2.38)$ & 2.76 & 0.0058 & 0.044 \\
\hline 0.02 & 1.19 & 0.57 & $(0.06-2.31)$ & 0.009 & 0.039 & 0.093 & 2.17 & 1.02 & $(0.17-4.18)$ & 0.01 & 0.03 & 0.088 & 1.42 & $(0.44-2.4)$ & 2.84 & 0.0045 & 0.044 \\
\hline 0.03 & 1.35 & 0.57 & $(0.22-2.48)$ & 0.012 & 0.02 & 0.068 & 1.95 & 1.02 & $(-0.04-3.95)$ & 0.008 & 0.06 & 0.108 & 1.49 & $(0.51-2.47)$ & 2.98 & 0.0029 & 0.035 \\
\hline 0.04 & 1.37 & 0.58 & $(0.23-2.51)$ & 0.012 & 0.019 & 0.068 & 1.94 & 1.02 & $(-0.06-3.95)$ & 0.008 & 0.06 & 0.109 & 1.51 & $(0.52-2.5)$ & 2.99 & 0.0028 & 0.035 \\
\hline 0.05 & 1.47 & 0.58 & $(0.33-2.62)$ & 0.014 & 0.012 & 0.057 & 2 & 1.02 & $(0-4.01)$ & 0.008 & 0.05 & 0.105 & 1.6 & $(0.61-2.6)$ & 3.17 & 0.0015 & 0.027 \\
\hline 0.1 & 1.55 & 0.58 & $(0.41-2.7)$ & 0.015 & 0.008 & 0.047 & 2.15 & 1.04 & $(0.12-4.18)$ & 0.009 & 0.04 & 0.093 & 1.7 & $(0.7-2.69)$ & 3.34 & 0.0008 & 0.024 \\
\hline 0.2 & 1.46 & 0.59 & $(0.31-2.62)$ & 0.013 & 0.013 & 0.057 & 2.12 & 1.03 & $(0.1-4.15)$ & 0.009 & 0.04 & 0.093 & 1.63 & $(0.62-2.63)$ & 3.17 & 0.0015 & 0.027 \\
\hline 0.3 & 1.46 & 0.59 & $(0.31-2.61)$ & 0.013 & 0.013 & 0.057 & 1.97 & 1.04 & $(-0.08-4)$ & 0.008 & 0.06 & 0.109 & 1.58 & $(0.58-2.59)$ & 3.1 & 0.0019 & 0.027 \\
\hline 0.4 & 1.45 & 0.58 & $(0.3-2.6)$ & 0.013 & 0.014 & 0.057 & 2.07 & 1.04 & $(0.02-4.12)$ & 0.008 & 0.05 & 0.103 & 1.6 & $(0.6-2.6)$ & 3.13 & 0.0017 & 0.027 \\
\hline 0.5 & 1.42 & 0.59 & $(0.27-2.57)$ & 0.012 & 0.016 & 0.058 & 2.12 & 1.04 & $(0.08-4.16)$ & 0.009 & 0.04 & 0.096 & 1.59 & $(0.59-2.59)$ & 3.11 & 0.0019 & 0.027 \\
\hline
\end{tabular}

PRS_PT $=$ P value threshold for ADHD polygenic risk score $\mathrm{R}^{2}=$ percentage of explained variance) ; FDR_P = false rate discovery corrected $\mathrm{P}$ for multiple testing $; 95 \% \mathrm{CI}=95 \%$ confidence intervals. 
Table 4.

ADHD-PRS association with linear WURS score (cADHD as a trait) in the total BP samples with BP age of onset as a covariate (linear regressions)

\begin{tabular}{|c|c|c|c|c|c|c|c|c|c|c|c|c|c|c|c|c|c|}
\hline \multirow{2}{*}{ PRS_PT } & \multicolumn{5}{|c|}{ Romanian Data } & \multirow[b]{2}{*}{ FDR_P } & \multicolumn{5}{|c|}{ UK Data } & \multirow[b]{2}{*}{ FDR_P } & \multicolumn{5}{|c|}{ Fixed Effect Meta-Analysis } \\
\hline & Beta & SE & $95 \% \mathrm{CI}$ & $\mathbf{R}^{2}$ & $\mathbf{P}$ & & Beta & SE & $95 \% \mathrm{CI}$ & $\mathbf{R}^{2}$ & $\mathbf{P}$ & & Beta & $95 \% \mathrm{CI}$ & $\mathbf{Z}$ & $\mathbf{P}$ & FDR_P \\
\hline 0.01 & 1.02 & 0.58 & $(-0.13-2.17)$ & 0.148 & 0.082 & 0.127 & 2.71 & 1.10 & $(0.55-4.86)$ & 0.047 & 0.014 & 0.058 & 1.4 & $(0.38-2.41)$ & 2.7 & 0.0069 & 0.044 \\
\hline 0.02 & 1.2 & 0.58 & $(0.07-2.34)$ & 0.151 & 0.038 & 0.093 & 2.35 & 1.09 & $(0.2-4.5)$ & 0.044 & $\mathbf{0 . 0 3 2}$ & 0.088 & 1.45 & $(0.45-2.46)$ & 2.84 & 0.0045 & 0.044 \\
\hline 0.03 & 1.39 & 0.58 & $(0.25-2.52)$ & 0.153 & 0.017 & 0.063 & 2.22 & 1.09 & $(0.08-4.35)$ & 0.042 & 0.043 & 0.096 & 1.57 & $(0.57-2.57)$ & 3.06 & 0.0022 & 0.029 \\
\hline 0.04 & 1.51 & 0.58 & $(0.36-2.65)$ & 0.155 & 0.01 & 0.054 & 2.25 & 1.09 & $(0.11-4.39)$ & 0.043 & 0.04 & 0.093 & 1.67 & $(0.66-2.68)$ & 3.24 & 0.0012 & 0.024 \\
\hline 0.05 & 1.53 & 0.58 & $(0.39-2.68)$ & 0.156 & 0.009 & 0.049 & 2.33 & 1.09 & $(0.19-4.47)$ & 0.043 & $\mathbf{0 . 0 3 3}$ & 0.088 & 1.71 & $(0.7-2.72)$ & 3.32 & 0.0009 & 0.024 \\
\hline 0.1 & 1.63 & 0.59 & $(0.47-2.78)$ & 0.157 & 0.006 & 0.044 & 2.8 & 1.11 & $(0.62-4.97)$ & 0.048 & 0.012 & 0.057 & 1.88 & $(0.86-2.9)$ & 3.62 & 0.0003 & 0.024 \\
\hline 0.2 & 1.59 & 0.59 & $(0.42-2.75)$ & 0.156 & 0.008 & 0.047 & 2.55 & 1.11 & $(0.38-4.73)$ & 0.045 & 0.022 & 0.072 & 1.8 & $(0.77-2.83)$ & 3.43 & 0.0006 & 0.024 \\
\hline 0.3 & 1.63 & 0.59 & $(0.47-2.79)$ & 0.157 & 0.0061 & 0.044 & 2.18 & 1.12 & $(-0.01-4.37)$ & 0.041 & 0.052 & 0.106 & 1.75 & $(0.73-2.77)$ & 3.35 & 0.0008 & 0.024 \\
\hline 0.4 & 1.64 & 0.59 & $(0.48-2.8)$ & 0.157 & 0.0057 & 0.044 & 2.14 & 1.12 & $(-0.06-4.33)$ & 0.041 & 0.057 & 0.109 & 1.75 & $(0.72-2.77)$ & 3.34 & 0.0008 & 0.024 \\
\hline 0.5 & 1.63 & 0.59 & $(0.47-2.79)$ & 0.157 & 0.0062 & 0.044 & 2.14 & 1.12 & $(-0.05-4.33)$ & 0.041 & 0.057 & 0.109 & 1.74 & $(0.71-2.76)$ & 3.32 & 0.0009 & 0.024 \\
\hline
\end{tabular}

PRS_PT $=$ P value threshold for ADHD polygenic risk score; $\mathrm{R}^{2}=$ percentage of explained variance); FDR_P = false rate discovery corrected $\mathrm{P}$ for multiple testing $; 95 \% \mathrm{CI}=95 \%$ confidence intervals. 
Table 5.

Prediction of cADHD (WURS score) in early onset BP cases (AO $\leq 21$ years) by ADHD PRS_PTs.

\begin{tabular}{|c|c|c|c|c|c|c|c|c|c|c|c|c|c|c|c|c|c|}
\hline \multirow[b]{2}{*}{ PRS_PT } & \multicolumn{5}{|c|}{ Romanian Data } & \multirow[b]{2}{*}{ FDR_P } & \multicolumn{5}{|c|}{ UK Data } & \multirow[b]{2}{*}{ FDR_P } & \multicolumn{5}{|c|}{ Fixed Effect Meta-Analysis } \\
\hline & Beta & SE & $95 \% \mathrm{Cl}$ & $\mathbf{R 2}$ & $\mathbf{P}$ & & Beta & SE & $95 \% \mathrm{Cl}$ & $\mathbf{R 2}$ & $\mathbf{P}$ & & $\begin{array}{l}\text { Bet } \\
\text { a }\end{array}$ & $95 \% \mathrm{Cl}$ & $\mathbf{Z}$ & $\mathbf{P}$ & FDR_P \\
\hline 0.01 & 1.28 & 0.86 & $(-0.39-2.96)$ & 0.009 & 0.13 & 0.187 & 3.51 & 1.52 & $(0.52-6.51)$ & 0.025 & 0.022 & 0.073 & 1.82 & $(0.35-3.28)$ & 2.43 & 0.015 & 0.032 \\
\hline 0.02 & 1.62 & 0.84 & $(-0.02-3.26)$ & 0.015 & 0.053 & 0.107 & 3.18 & 1.53 & $(0.18-6.18)$ & 0.02 & 0.039 & 0.093 & 1.98 & $(0.54-3.42)$ & 2.7 & 0.007 & $\mathbf{0 . 0 2 0}$ \\
\hline 0.03 & 1.8 & 0.83 & $(0.18-3.42)$ & 0.019 & 0.031 & 0.086 & 2.79 & 1.55 & $(-0.25-5.83)$ & 0.015 & 0.074 & 0.119 & 2.02 & $(0.59-3.45)$ & 2.77 & 0.0057 & $\mathbf{0 . 0 1 7}$ \\
\hline 0.04 & 1.93 & 0.84 & $(0.29-3.58)$ & 0.021 & 0.022 & 0.072 & 2.61 & 1.56 & $(-0.46-5.68)$ & 0.013 & 0.097 & 0.145 & 2.08 & $(0.63-3.53)$ & 2.82 & 0.0049 & 0.016 \\
\hline 0.1 & 2.11 & 0.85 & $(0.45-3.78)$ & 0.025 & 0.014 & 0.057 & 3.57 & 1.55 & $(0.53-6.6)$ & 0.025 & 0.022 & 0.073 & 2.45 & $(0.99-3.91)$ & 3.29 & 0.001 & 0.007 \\
\hline 0.2 & 2.12 & 0.86 & $(0.44-3.8)$ & 0.025 & 0.014 & 0.058 & 3.73 & 1.52 & $(0.74-6.72)$ & 0.028 & 0.015 & 0.058 & 2.51 & $(1.04-3.97)$ & 3.36 & 0.0008 & 0.007 \\
\hline 0.3 & 2.11 & 0.84 & $(0.47-3.75)$ & 0.025 & 0.013 & 0.057 & 3.24 & 1.52 & $(0.26-6.23)$ & 0.021 & 0.035 & 0.088 & 2.37 & $(0.93-3.81)$ & 3.23 & 0.0012 & 0.007 \\
\hline 0.4 & 2.17 & 0.84 & $(0.52-3.81)$ & 0.027 & 0.01 & 0.054 & 3.18 & 1.53 & $(0.18-6.17)$ & 0.02 & 0.039 & 0.093 & 2.4 & $(0.96-3.84)$ & 3.26 & 0.0011 & 0.007 \\
\hline 0.5 & 2.15 & 0.84 & $(0.5-3.79)$ & 0.026 & 0.011 & 0.056 & 3.13 & 1.52 & $(0.15-6.1)$ & 0.02 & 0.041 & 0.093 & 2.38 & $(0.94-3.81)$ & 3.24 & 0.0012 & 0.007 \\
\hline
\end{tabular}

PRS_PT = P value threshold for ADHD polygenic risk score; $\mathrm{R}^{2}=$ percentage of explained variance); FDR_P = false rate discovery corrected $\mathrm{P}$ for multiple testing $; 95 \% \mathrm{CI}=95 \%$ confidence intervals 


\section{Table 6.}

Differentiation of early onset BP cases from controls (logistic regression with the dependent variable "affected - not affected with BP" and ten ADHD-

PRS-PTs as predictors).

\begin{tabular}{|c|c|c|c|c|c|c|c|c|c|c|c|c|c|c|c|c|c|}
\hline \multirow{2}{*}{ PRS_PT } & \multicolumn{5}{|c|}{ Romanian Data } & \multirow[b]{2}{*}{ FDR_P } & \multicolumn{5}{|c|}{ UK Data } & \multirow[b]{2}{*}{ FDR_P } & \multicolumn{5}{|c|}{ Fixed Effect Meta-Analysis } \\
\hline & OR & SE & $95 \% \mathrm{Cl}$ & $\mathbf{R}^{2}$ & $\mathbf{P}$ & & OR & SE & $95 \% \mathrm{Cl}$ & $\mathbf{R}^{2}$ & $\mathbf{P}$ & & OR & $95 \% \mathrm{Cl}$ & $\mathbf{Z}$ & $\mathbf{P}$ & FDR_P \\
\hline 0.01 & 1.28 & 0.86 & $-0.39-2.96$ & 0.009 & 0.13 & 0.19 & 3.51 & 1.53 & $0.52-6.51$ & 0.025 & 0.022 & 0.073 & 1.82 & $0.35-3.28$ & 2.43 & 0.015 & 0.058 \\
\hline 0.02 & 1.62 & 0.84 & $-0.02-3.26$ & 0.015 & 0.053 & 0.11 & 3.18 & 1.53 & $0.18-6.18$ & 0.02 & 0.039 & 0.094 & 1.98 & $0.54-3.42$ & 2.7 & 0.007 & 0.045 \\
\hline 0.03 & 1.8 & 0.83 & $0.18-3.42$ & 0.019 & 0.031 & 0.086 & 2.79 & 1.55 & $-0.25-5.83$ & 0.015 & 0.074 & 0.12 & 2.02 & $0.59-3.45$ & 2.77 & 0.0057 & 0.045 \\
\hline 0.04 & 1.93 & 0.84 & $0.29-3.58$ & 0.021 & 0.022 & 0.073 & 2.61 & 1.57 & $-0.46-5.68$ & 0.013 & 0.097 & 0.15 & 2.08 & $0.63-3.53$ & 2.82 & 0.0049 & 0.045 \\
\hline 0.05 & 1.91 & 0.85 & $0.24-3.58$ & 0.02 & 0.026 & 0.079 & 2.79 & 1.55 & $-0.26-5.83$ & 0.015 & 0.074 & 0.12 & 2.11 & $0.65-3.58$ & 2.82 & 0.0047 & 0.045 \\
\hline 0.1 & 2.11 & 0.85 & $0.45-3.78$ & 0.025 & 0.014 & 0.058 & 3.57 & 1.55 & $0.53-6.6$ & 0.025 & 0.022 & 0.073 & 2.45 & $0.99-3.91$ & 3.29 & 0.001 & 0.024 \\
\hline 0.2 & 2.12 & 0.86 & $0.44-3.8$ & 0.025 & 0.014 & 0.058 & 3.73 & 1.53 & $0.74-6.72$ & 0.028 & 0.015 & 0.058 & 2.51 & $1.04-3.97$ & 3.36 & 0.0008 & 0.024 \\
\hline 0.3 & 2.11 & 0.84 & $0.47-3.75$ & 0.025 & 0.013 & 0.057 & 3.24 & 1.52 & $0.26-6.23$ & 0.021 & 0.035 & 0.089 & 2.37 & $0.93-3.81$ & 3.23 & 0.0012 & 0.024 \\
\hline 0.4 & 2.17 & 0.84 & $0.52-3.81$ & 0.027 & 0.01 & 0.055 & 3.18 & 1.53 & $0.18-6.17$ & 0.02 & 0.039 & 0.094 & 2.4 & $0.96-3.84$ & 3.26 & 0.0011 & 0.024 \\
\hline 0.5 & 2.15 & 0.84 & $0.5-3.79$ & 0.026 & 0.011 & 0.056 & 3.13 & 1.52 & $0.15-6.1$ & 0.02 & 0.041 & 0.094 & 2.38 & $0.94-3.81$ & 3.24 & 0.0012 & 0.024 \\
\hline
\end{tabular}

PRS_PT $=$ P value threshold for ADHD polygenic risk score; $\mathrm{R}^{2}=$ Nagelkerke $\mathrm{R}^{2}$ (percentage of explained variance); FDR_P $=$ false rate discovery corrected P for multiple testing 